\title{
Qualidade da água de abastecimento público de Ribeirão Preto em área de abrangência do Aquífero Guarani: determinação de metais e nitrato
}

(doi:10.4136/ambi-agua.148)

\author{
Sérgio Marcos Sanches; Eny Maria Vieira²; Eliana Leão do Prado³; Angela Maria \\ Magosso Takayanagui ${ }^{4}$ \\ ${ }^{1}$ Instituto Federal de Educação, Ciência e Tecnologia Triângulo Mineiro - IFTM - Campus Ituiutaba - MG \\ E-mail: sergiosanches@iftriangulo.edu.br \\ ${ }^{2}$ Instituto de Química de São Carlos - USP \\ E-mail: eny@iqsc.usp.br \\ ${ }^{3}$ Escola de Enfermagem de Ribeirão Preto - USP \\ E-mail: leprado@ig.com.br \\ ${ }^{4}$ Escola de Enfermagem de Ribeirão Preto - USP \\ E-mail: ammtakay@eerp.usp.br
}

\section{RESUMO}

A cidade de Ribeirão Preto-SP é totalmente abastecida por água subterrânea, proveniente do Aquífero Guarani. A cidade possui um total de 102 poços de abastecimento público cadastrados no Departamento de Água Esgoto de Ribeirão Preto - Daerp, de onde são captados aproximadamente $13400 \mathrm{~m}^{3} / \mathrm{h}$ de água. Alguns poços estão localizados, na área de recarga do Aquífero Guarani, região vulnerável à poluição antrópica. Este estudo objetivou avaliar a qualidade da água de poços de abastecimento público de Ribeirão Preto-SP quanto a alguns parâmetros químicos, como $\mathrm{pH}$, metais e nitrato. Foram feitas três coletas em 33 poços de água de abastecimento público, nos meses de maio e novembro de 2008, e também em julho de 2009. As análises dos metais Fe, Zn, Mn, Cr, Pb, Cu e Cd foram feitas utilizando-se a técnica de Espectroscopia com Plasma Induzido (ICP-OES); a análise de nitrato foi feita pelo método espectrofotométrico. Quanto aos metais e ao nitrato, todas as amostras apresentaramse dentro dos valores estabelecidos pela Portaria do Ministério da Saúde $n^{\circ} 518$ de 2004 . Em relação ao $\mathrm{pH}$, um número pequeno de poços de abastecimento apresentaram $\mathrm{pH}$ fora do limite de potabilidade, embora com valores muito próximos do limite inferior estabelecido pela referida portaria. Os resultados revelaram que a água dos poços de abastecimento público de Ribeirão Preto - SP, analisados neste estudo, apresentaram de uma forma geral, uma qualidade adequada, sem restrições ao uso. Porém, remetem à necessidade de manutenção de um contínuo monitoramento de todos os poços de abastecimento público, especialmente quanto à presença de nitrato e cromo, segundo relatório da Cetesb.

Palavras-chave: Qualidade da água para abastecimento público; parâmetros químicos; Aquifero Guarani.

\section{Quality of public water supply of Ribeirão Preto in the Guarani aquifer area: metals and nitrate analyses}

\section{ABSTRACT}

The city of Ribeirão Preto, SP, is fully supplied by groundwater from the Guarani aquifer. The city has a total of 102 public supply wells registered in the Ribeirão Preto Water and Sewage Department, approximately $13,400 \mathrm{~m}^{3} / \mathrm{h}$ of water are uptaken from them. Some wells are located in the recharge area of the Guarani Aquifer, a region susceptible to anthropogenic pollution. This study aimed to evaluate the quality of water of public supply 
SANCHES, S. M.; VIEIRA, E. M.; PRADO, E. L.; TAKAYANAGUI, A. M. M. Qualidade da água de abastecimento público de Ribeirão Preto em área de abrangência do Aquífero Guarani: determinação de metais e nitrato. Ambi-Agua, Taubaté, v. 5, n. 2, p. 202-216, 2010. (doi:10.4136/ambi-agua.148)

wells in Ribeirão Preto considering specific chemical parameters including $\mathrm{pH}$, metals and nitrate. Three sampling collections were performed in 33 wells for water supply in Ribeirão Preto, in May and November 2008, and in July 2009, as well. Analyses of the metals Fe, Zn, $\mathrm{Mn}, \mathrm{Cr}, \mathrm{Pb}, \mathrm{Cu}$ and $\mathrm{Cd}$ were performed using the Induced Plasma Spectroscopy technique (ICP-OES). The analyses of nitrate concentration were performed using spectrophotometric method. Regarding the metals and nitrate parameters, all samples were within the values established by the ordinance of the Ministry of Health No. 518 of 2004. Regarding pH, a small number of wells had $\mathrm{pH}$ below the limit of drinkable water, but values were very close to the lower limit established by the ordinance. Water of public supply wells in Ribeirão Preto are, in general, of good quality, showing no restrictions for use. Nevertheless, continuous monitoring of all public supply wells is necessary, especially regarding the presence of nitrate and chromium, second report of Company of Technology and Environmental Sanitation Cetesb.

Keywords: Quality of public water supply; chemical parameters; Guarani aquifer.

\section{INTRODUÇÃO}

A água é uma substância de fundamental importância para a manutenção da vida no Planeta, constituindo-se no elemento inorgânico mais abundante na matéria viva, como 60\% do peso corporal humano e percentual mais elevado em certos animais (Rebouças, 2006). Do total de água existente no Planeta, $97 \%$ são provenientes dos oceanos e 3\% de água doce; desses 3\%, 2\% encontram-se em geleiras, 0,7\% em lençóis subterrâneos e 0,3\% em lagos (Rebouças, 2006).

A água própria para o consumo humano é, portanto, escassa, e a degradação desse recurso pelo homem vem agravando as dificuldades para seu aproveitamento.

A água subterrânea constitui-se em uma importante fonte de água destinada ao abastecimento público de algumas cidades brasileiras, captadas dos aquíferos por meio de poços que podem ser tubulares ou em cacimba. Dentre os principais aquíferos destaca-se o Guarani, que se localiza em quatro territórios, Brasil, Argentina, Paraguai e Uruguai. Sua área é estimada em 1,2 milhões de quilômetros quadrados, abrigando um volume de 45 mil quilômetros cúbicos de água (Gastamans e Kiang, 2005). Sua maior ocorrência se dá em território brasileiro (2/3 da área total), abrangendo os Estados de Goiás, Mato Grosso do Sul, Minas Gerais, Paraná, Santa Catarina, Rio Grande do Sul e São Paulo (Borghetti et al., 2004).

Segundo dados da Companhia de Tecnologia e Saneamento Ambiental - Cetesb, órgão do Estado de São Paulo responsável pelo controle ambiental, em 1997, cerca de $72 \%$ dos municípios paulistas utilizavam água subterrânea no abastecimento público (Iritani e Ezaqui, 2008). Em 2006, essa porcentagem subiu para 80\%.

A cidade de Ribeirão Preto - SP é totalmente abastecida por água subterrânea, e possui 102 poços cadastrados no Departamento de Água e Esgoto - Daerp. A principal vantagem da utilização da água subterrânea é que ela é uma fonte mais segura e menos onerosa (Eckhard et al., 2009); porém, em Ribeirão Preto, alguns poços estão localizados em área de recarga do Aquífero Guarani, região vulnerável à poluição. Entende-se por vulnerabilidade o conjunto das características do aquifero que determina o quanto ele poderá ser afetado pela ação de determinado poluente (Silva, 2003). Os fatores que determinam a vulnerabilidade do aquífero são: a espessura da zona não saturada, tipo de porosidade, tipo de rocha ou solo, velocidade das águas subterrâneas e o rendimento do aquífero. O poluente será mais perigoso quanto mais móvel e persistente for aos meios saturados e não-saturados. As principais fontes de poluição antrópicas sobre a qualidade da água são: a presença de fossas sépticas, depósito de lixo, vazamento de rede de esgotos, fertilizantes, e pesticidas empregados na agricultura (Iritani e Ezaki, 2008). 
SANCHES, S. M.; VIEIRA, E. M.; PRADO, E. L.; TAKAYANAGUI, A. M. M. Qualidade da água de abastecimento público de Ribeirão Preto em área de abrangência do Aquífero Guarani: determinação de metais e nitrato. Ambi-Agua, Taubaté, v. 5, n. 2, p. 202-216, 2010. (doi:10.4136/ambi-agua.148)

Segundo Rebouças (1992), as alterações na qualidade da água subterrânea são ditas diretas quando engendradas por substâncias naturais ou artificiais introduzidas pelo homem no ciclo geoquímico da Terra. Influências indiretas podem ser consideradas como alterações da qualidade, as quais ocorrem sem adição das substâncias geradas pelo homem ou são produzidas pela interferência nos processos químicos, físicos, biológicos e hidrológicos.

Dentre os diferentes contaminantes químicos, o estudo dos metais e nitrato vem sendo considerado, mundialmente, como prioritário nos programas de promoção da saúde, visto que dependendo da dose e da forma química em que se encontram, podem ocasionar sérios danos ao sistema biológico dos seres humanos, ocasionando o aparecimento de doenças (Celere et al., 2007).

Os metais pesados, elementos de elevada massa molecular, quando absorvidos pelo ser humano são acumulativos, e se depositam no tecido ósseo e gorduroso ocasionando o deslocamento de minerais nobres dos ossos e músculos para a circulação. Esse processo pode provocar doenças tais como anemia, doença renal, distúrbios na reprodução e danos neurológicos (Tonani, 2008).

Portanto, diante do exposto, o objetivo desta pesquisa foi o de verificar a qualidade da água de poços de abastecimento público de Ribeirão Preto - SP, quanto ao $\mathrm{pH}$, nitrato e metais.

\section{MATERIAL E MÉTODOS}

\subsection{Localizações dos pontos de coleta de água dos poços de abastecimento público de Ribeirão Preto-SP}

De acordo com o Departamento de Água e Esgoto de Ribeirão Preto- SP- Daerp, a cidade possui 102 poços de abastecimento público cadastrados e em funcionamento (Ribeirão Preto, 2007). Para este estudo foram coletadas amostras de água em 33 poços de abastecimento público de Ribeirão Preto - SP durante 3 períodos, 28-05-2008, 25-11-2008 e 30-07-2009.

Essa amostragem corresponde a 32,35\% do total de poços existentes. Os pontos de coleta foram selecionados de forma a abranger proporcionalmente as cinco regiões do município (Norte, Sul, Leste, Oeste e Central), partindo-se de um cadastro disponibilizado pelo Daerp. Os pontos de coleta foram decididos em comum acordo com a chefia do Laboratório de análises Química do Daerp, conforme Tabela 1 e Figura 1, a seguir.

\subsection{Amostragem de água para as análises de metais, nitrato e p $\mathbf{H}$}

Coletaram-se três amostras de água de cada poço de abastecimento público selecionado. Uma amostra foi utilizada para a determinação de metais, a outra, para determinação de $\mathrm{pH}$ e a última, para a determinação de nitrato. Durante a coleta das amostras de água, as torneiras dos poços selecionados foram abertas e deixadas escoar por 5 minutos. Em seguida, os frascos foram abertos e ambientados com água do local, para minimizar possíveis interferências. Após essa etapa, coletaram-se as amostras de água em frascos, que foram mantidas em caixa de isopor, com gelo, até o final da coleta. As amostras destinadas para análise de metais foram filtradas e acidificadas com ácido nítrico (suprapuro) até $\mathrm{pH} 2$, sendo armazenadas em geladeira, à temperatura de $4^{\circ} \mathrm{C}$, no Laboratório de Saúde Ambiental da Escola de Enfermagem de Ribeirão Preto - SP/USP, para análise posterior.

\subsection{Determinação de $\mathrm{pH}$ das amostras de água coletadas nos poços de abastecimento público}

As análises de $\mathrm{pH}$ foram feitas na Central de Análise Químicas do Instituto de Química do Campus da USP de São Carlos - SP, utilizando-se um pHmetro da Marca Orion 710-A, previamente calibrado com os tampões de $\mathrm{pH} 4,7$ e 10 . Todas as medidas foram feitas à temperatura de $25^{\circ} \mathrm{C}$. 
SANCHES, S. M.; VIEIRA, E. M.; PRADO, E. L.; TAKAYANAGUI, A. M. M. Qualidade da água de abastecimento público de Ribeirão Preto em área de abrangência do Aquífero Guarani: determinação de metais e nitrato. Ambi-Agua, Taubaté, v. 5, n. 2, p. 202-216, 2010. (doi:10.4136/ambi-agua.148)

Tabela 1. Localizações dos pontos de coleta das amostras de água de poços de abastecimento público de Ribeirão Preto - SP.

\begin{tabular}{|c|c|c|}
\hline \multirow{2}{*}{$\begin{array}{l}\text { Poços de água de } \\
\text { abastecimento público } \\
\text { de Ribeirão Preto - SP }\end{array}$} & \multicolumn{2}{|c|}{ Coordenadas UTM (m) } \\
\hline & $\mathbf{N}$ & $\mathbf{E}$ \\
\hline 1 & 7.646 .757 & 208.513 \\
\hline 2 & 7.657 .523 & 208.011 \\
\hline 3 & 7.656 .684 & 205.290 \\
\hline 4 & 7.656 .954 & 207.813 \\
\hline 5 & 7.655 .846 & 207.320 \\
\hline 6 & 7.658 .180 & 208.470 \\
\hline 7 & 7.656 .646 & 206.616 \\
\hline 8 & 7.661 .468 & 210.783 \\
\hline 9 & 7.653 .662 & 204.883 \\
\hline 10 & 7.651 .743 & 207.303 \\
\hline 11 & 7.656.176 & 205.073 \\
\hline 12 & 7.661 .564 & 207.094 \\
\hline 13 & 7.659 .873 & 210.198 \\
\hline 14 & 7.655 .781 & 205.838 \\
\hline 15 & 7.652 .201 & 210.091 \\
\hline 16 & 7.654 .435 & 206.693 \\
\hline 17 & 7.655 .480 & 205.888 \\
\hline 18 & 7.663 .243 & 210.788 \\
\hline 19 & 7.648 .215 & 206.332 \\
\hline 20 & 7.660 .342 & 203.901 \\
\hline 21 & 7.661 .966 & 209.800 \\
\hline 22 & 7.657 .369 & 204.473 \\
\hline 23 & 7.654 .779 & 204.926 \\
\hline 24 & 7.654 .109 & 213.627 \\
\hline 25 & 7.655 .084 & 212.854 \\
\hline 26 & 7.660 .807 & 215.854 \\
\hline 27 & 7.658 .231 & 210.103 \\
\hline 28 & 7.662 .620 & 208.860 \\
\hline 29 & 7.657 .950 & 208.250 \\
\hline 30 & 7.655 .623 & 215.883 \\
\hline 31 & 7.663 .454 & 208.603 \\
\hline 32 & 7.654.327 & 214.010 \\
\hline 33 & 7.658.937 & 203.586 \\
\hline
\end{tabular}

\subsection{Construção da curva analítica para as determinações de nitrato em água de abastecimento público}

A curva analítica foi feita com 7 pontos em triplicata. As concentrações utilizadas foram: 0,$00 ; 1,00 ; 2,00 ; 3,00 ; 4,00 ; 6,00$ e 7,00 $\mathrm{mg} \mathrm{L}^{-1}$. Na Figura 2, encontra-se a curva analítica para as determinações de nitrato. 
SANCHES, S. M.; VIEIRA, E. M.; PRADO, E. L.; TAKAYANAGUI, A. M. M. Qualidade da água de abastecimento público de Ribeirão Preto em área de abrangência do Aquífero Guarani: determinação de metais e nitrato. Ambi-Agua, Taubaté, v. 5, n. 2, p. 202-216, 2010. (doi:10.4136/ambi-agua.148)

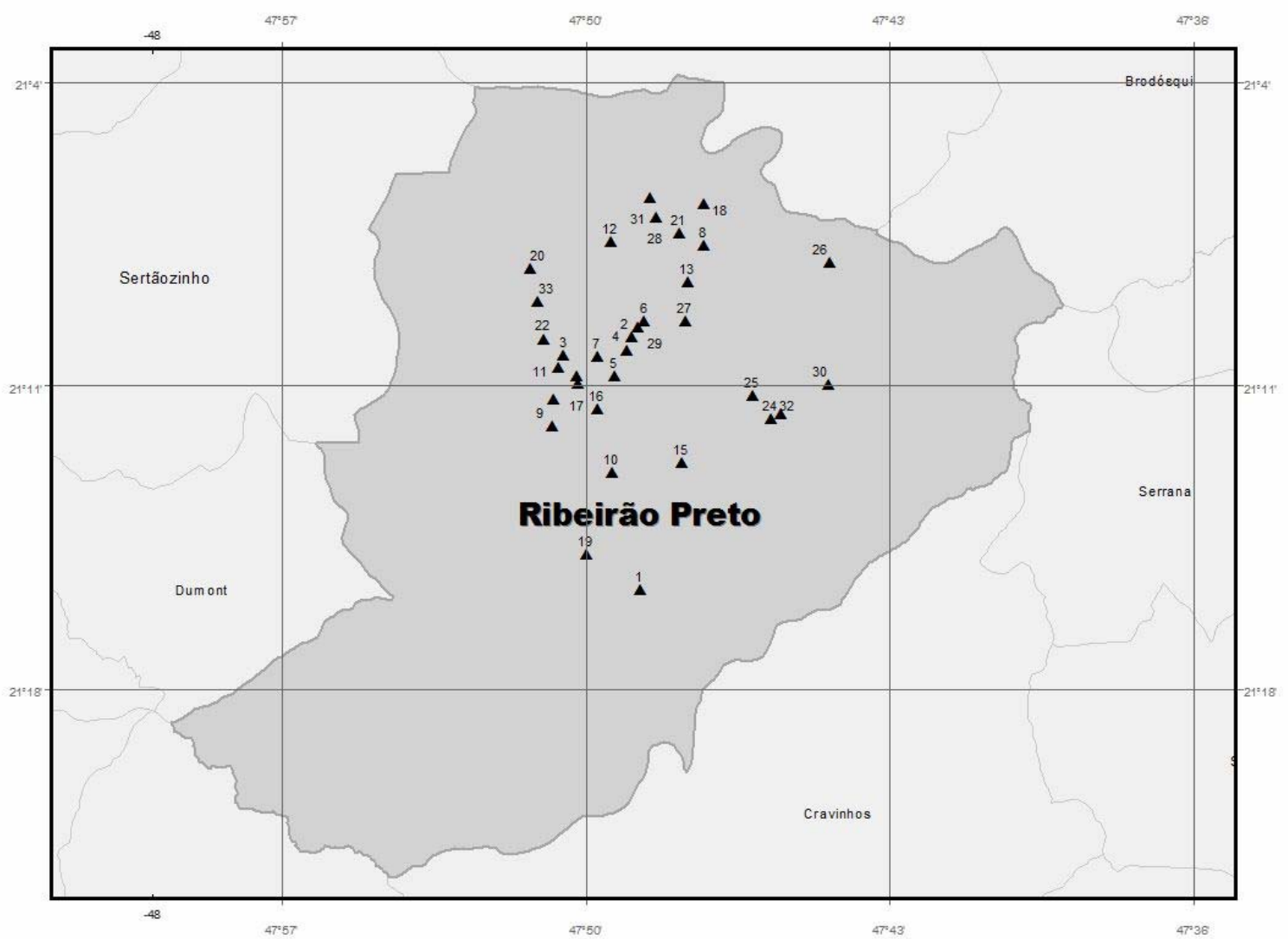

Figura 1. Localização dos 33 pontos de coleta de água dos poços de abastecimento público de Ribeirão Preto-SP.

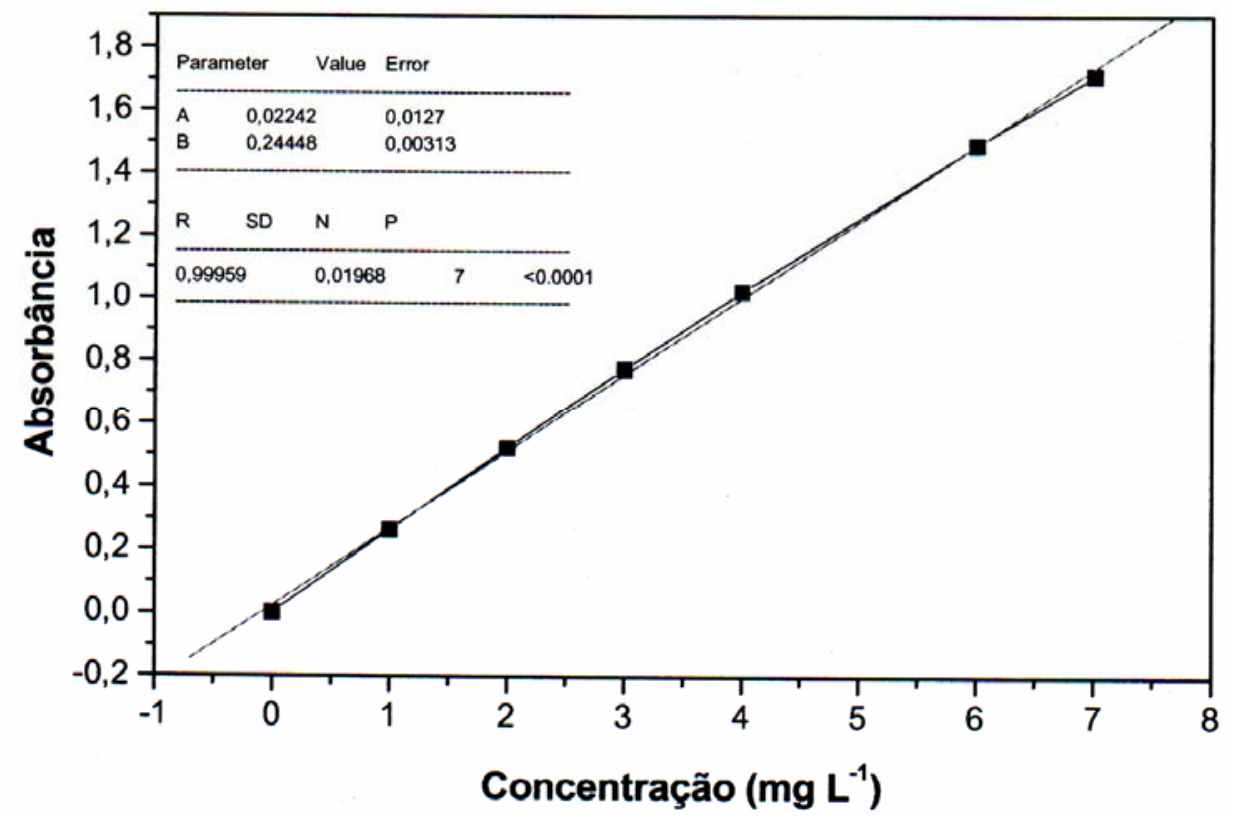

Figura 2. Curva analítica utilizada para a determinação de nitrato em água de poços de abastecimento público de Ribeirão Preto - SP.

\subsection{Determinação de nitrato}

As análises de nitrato foram feitas no Laboratório de Saneamento da Escola de Engenharia de São Carlos - EESC- USP, em um Espectrofotômetro de UV-Visível, Marca SHIMADZU, modelo UV 160 A. As leituras das amostras e do branco foram realizadas a 220 $\mathrm{nm}$. 


\subsection{Digestão das amostras de água para a determinação de metais em água de abastecimento público}

Para a realização da digestão das amostras de água para a determinação de metais, transferiram-se alíquotas de $100 \mathrm{~mL}$ de água, medida em balão volumétrico, para os tubos do bloco digestor, aos quais foram adicionados $2 \mathrm{~mL}$ de $\mathrm{HNO}_{3}$ (suprapuro) e $5 \mathrm{~mL}$ de $\mathrm{HCl}$ (suprapuro). Elevou-se a temperatura a $95^{\circ} \mathrm{C}$ e manteve-se o aquecimento até que o volume da amostra fosse reduzido a $15 \mathrm{~mL}$, sob refluxo. Após resfriamento, as amostras foram transferidas para um balão volumétrico de $50 \mathrm{~mL}$, completando-se então seu volume com água livre de compostos orgânicos. A amostra foi filtrada e mantida a $4^{\circ} \mathrm{C}$ até a análise. Esse método segue o procedimento do método 3005A da Environmental Protection Agency (Usepa, 1992) para análise de metais.

\subsection{Construção das curvas analíticas para a determinação de metais em água de abastecimento público de Ribeirão Preto - SP}

As curvas analíticas para a determinação de metais foram preparadas com 3 pontos mais o branco, sendo cada ponto preparado em triplicada, a partir de solução estoque, de concentração de $1000 \mathrm{mg} \mathrm{L}^{-1}$. As concentrações utilizadas para os metais $\mathrm{Fe}, \mathrm{Zn}, \mathrm{Cd}, \mathrm{Cr}, \mathrm{Pb}$, $\mathrm{Cu}$ foram:

$$
\begin{aligned}
& \text { Fe - 0,00; 0,20; 0,40 e 0,80 mg L }{ }^{-1} \mathrm{Zn}-0,00 ; 3,00 ; 5,00 \text { e } 7,00 \mathrm{mg} \mathrm{L}^{-1} \\
& \mathrm{Cd}-0,00 ; 0,004 ; 0,03 \text { e } 0,06 \mathrm{mg} \mathrm{L}^{-1} . \mathrm{Cr}-0,00 ; 0,04 ; 0,008 \text { e } 0,120 \mathrm{mg} \mathrm{L}^{-1} \\
& \mathrm{~Pb}-0,00 ; 0,030 ; 0,060 \text { e } 0,090 \mathrm{mg} \mathrm{L}^{-1} \mathrm{Cu}-0,00 ; 1,00 ; 2,00 \text { e } 3,00 \mathrm{mg} \mathrm{L}^{-1}
\end{aligned}
$$

\subsection{Determinações de metais em água de abastecimento público de Ribeirão Preto - SP}

A análise dos metais $\mathrm{Fe}, \mathrm{Zn}, \mathrm{Mn}, \mathrm{Cr}, \mathrm{Pb}, \mathrm{Cu}$ e Cd foram feitas na Central de Análise Químicas do Instituto de Química do Campus da USP de São Carlos - SP, em um equipamento de Espectroscopia com Plasma Induzido (ICP-OES), marca PERKIN ELMER, modelo Optima 3000 DV. Os comprimentos de onda utilizados nas análises de metais, e os limites de detecção estão na Tabela 2.

Tabela 2. Comprimentos de onda utilizados nas análises de metais e os limites de detecção.

\begin{tabular}{l|c|c}
\hline Metais & $\begin{array}{c}\text { Comprimento de onda } \\
(\mathbf{n m})\end{array}$ & $\begin{array}{c}\text { Limite de detecção } \\
\left(\mathbf{\mu g} \mathbf{L}^{-\mathbf{1}}\right)\end{array}$ \\
\hline Cromo & 205,560 & $0,1-1,0$ \\
Manganês & 257,610 & $0,1-1,0$ \\
Cádmio & 214,440 & $<0,1$ \\
Cobre & 324,752 & $0,1-1,0$ \\
Zinco & 206,200 & $0,1-1,0^{-}$ \\
Ferro & 238,204 & $<0,1$ \\
Chumbo & 220,353 & $1,0-10,0$ \\
\hline
\end{tabular}

\section{RESULTADOS E DISCUSSÃO}

\section{1. $\mathrm{pH}$}

O potencial hidrogeniônico é usado universalmente para expressar o grau de acidez ou basicidade de uma solução, ou seja, é o modo de expressar a concentração de íons de hidrogênio nessa solução. A escala de $\mathrm{pH}$ é constituída por uma série de números variando de 0 a 14, os quais denotam graus de acidez ou alcalinidade. Valores abaixo de 7 e próximos de zero indicam aumento de acidez, enquanto valores de 7 a 14 indicam aumento da basicidade. 
SANCHES, S. M.; VIEIRA, E. M.; PRADO, E. L.; TAKAYANAGUI, A. M. M. Qualidade da água de abastecimento público de Ribeirão Preto em área de abrangência do Aquífero Guarani: determinação de metais e nitrato. Ambi-Agua, Taubaté, v. 5, n. 2, p. 202-216, 2010. (doi:10.4136/ambi-agua.148)

Na Tabela 3, são apresentados os valores de pH das amostras de água coletadas em 33 poços de abastecimento público de Ribeirão Preto - SP, nos três momentos de coleta.

Analisando-se os dados apresentados na Tabela 3, nota-se que todas as amostras de água apresentaram um $\mathrm{pH}$ levemente ácido, devido os poços estarem localizados em região que possui condições freáticas, pois a porção aflorante do aquífero Guarani está localizada na região de Ribeirão Preto - SP. O pH encontrado passa a receber influência do gás carbônico proveniente das águas atmosféricas, e das raízes das plantas. Segundo Borghetti et al. (2004), em termos de potabilidade, as águas dos basaltos revelam uma forte tendência a tornar-se alcalina, pH entre 5,5 e 6,5 (Eckhardt et al., 2009). Dos 33 poços de água de abastecimento público de Ribeirão Preto - SP, analisados no dia 28-05-2008, 12,12\% apresentaram pH fora do limite de potabilidade, cujos valores são estabelecidos pela Portaria 518 do Ministério da Saúde de 2004, que determina que para a água de consumo humano, os valores devem permanecer na faixa de 6,0 a 9,5 (Brasil, 2004). Nas coletas feitas nos dias 25-11-2008 e 3007-2009, 5 poços apresentaram $\mathrm{pH}$ fora do limite de potabilidade. Comparando-se os valores de $\mathrm{pH}$ entre as três coletas feitas num mesmo poço, observa-se que ocorreu uma pequena variação nos valores de pH. Segundo Gastmans e Kiang (2005), as águas subterrâneas naturais são do tipo bicarbonatadas cálcicas-sódicas, neutras ( $\mathrm{pH}$ médio de 7) e pouco mineralizadas; porém, as águas provenientes das áreas com alto risco de contaminação são ácidas a levemente ácidas (pH médio de 6,5).

\subsection{Nitrato}

Os nitratos são uma das maiores fontes de íons naturais das águas e são obtidos a partir da oxidação da amônia pela ação das bactérias (nitrossomas). Ele é o constituinte inorgânico mais problemático, devido a uma ampla distribuição, grande mobilidade em sub-superfície, estabilidade em sistemas aeróbicos de águas subterrâneas e risco à saúde humana, principalmente em crianças e idosos (Eckhart et al., 2009). O seu consumo por meio de água de abastecimento está associado a dois efeitos adversos à saúde: a indução a metemoglobinemia, especialmente em crianças, e a formação potencial de nitrosaminas carcinogênicas (Alaburda e Nishiharala, 1998; Macêdo, 2004).

Quando o nitrato infiltra na camada do lençol freático e entra em contato com o oxigênio, a possibilidade de eliminação da substância é mínima (Macêdo, 2004). Na Tabela 4, estão os resultados das concentrações de nitrato em água de 33 poços de abastecimento púbico de Ribeirão - SP, em diferentes momentos da coleta.

Analisando-se os dados na Tabela 4 para a água coletada nos 33 poços de água de abastecimento público, nota-se que a concentração ficou na faixa de 0,0001 a 3,9505 $\mathrm{mg} \mathrm{L}^{-1}$. Esses valores indicam que todas as amostras analisadas encontram-se abaixo dos valores máximos permitidos pela Port MS nº 518/2004, e também abaixo do valor máximo orientado pela CETESB para águas subterrâneas do Estado de São Paulo, cujo valor é de $10 \mathrm{mg} \mathrm{L}^{-1}$.

Dentre os poços analisados o $\mathrm{n}^{\circ} 2$ e 4 apresentaram as maiores concentrações de nitrato, que foram respectivamente de 3,6326 e 3,9505 $\mathrm{mg} \mathrm{L}^{-1}$. Segundo Bouchard e William (1992), valores acima de $3,0 \mathrm{mg} \mathrm{L}^{-1}$ são indicativos de contaminação devido às atividades antropogênicas. As principais fontes de nitrato nas águas subterrâneas de origem antrópica são difusas, destacando-se a aplicação de fertilizantes e insumos nitrogenados, utilização de fossas negras, vazamentos das redes coletoras de esgoto e influência de rios contaminados na zona de captação de poços (Cetesb, 2001). Outra significante fonte de contaminação na região de Ribeirão Preto-SP pode ser pela utilização descontrolada de vinhoto no solo. Segundo Relatório de Qualidade das Águas Subterrâneas do Estado de São Paulo, publicado pela Cetesb entre 2004 e 2006, há uma tendência no aumento da concentração de nitrogênio nitrato (N - Nitrato). Comparando os resultados de nitrato obtidos pela Cetesb em poços monitorados no Aquífero Guarani em 2004, com os resultados apresentados na Tabela 2, há uma tendência do aumento da concentração de nitrato. Embora a concentração de nitrato não 
SANCHES, S. M.; VIEIRA, E. M.; PRADO, E. L.; TAKAYANAGUI, A. M. M. Qualidade da água de abastecimento público de Ribeirão Preto em área de abrangência do Aquífero Guarani: determinação de metais e nitrato. Ambi-Agua, Taubaté, v. 5, n. 2, p. 202-216, 2010. (doi:10.4136/ambi-agua.148)

tenha atingido o nível de tolerância do organismo humano, de $10 \mathrm{mg} \mathrm{L}^{-1}$. A Cetesb recomenda cuidados aos municípios no gerenciamento da qualidade da água. Na coleta realizada em 42 pontos de captação de água no Aquífero pela Cetesb, houve oscilação de 0,0001 e 11,7 mg L ${ }^{-1}$ de nitrato com média geral de $0,2 \mathrm{mg} \mathrm{L}^{-1}$. Do total de pontos monitorados, 15 foram na região de Araraquara. Em Ribeirão Bonito, foi encontrada concentração de nitrato acima de 5,0 mg $\mathrm{L}^{-1}$, considerado nível de atenção segundo índice da Cetesb, o que não ocorreu nesta investigação.

Tabela 3. Valores de pH medidos na água de poços de abastecimento público da cidade de Ribeirão Preto - SP.

\begin{tabular}{|c|c|c|c|}
\hline \multirow{2}{*}{$\begin{array}{l}\text { Poços de água de abastecimento } \\
\text { público de Ribeirão Preto - SP }\end{array}$} & \multicolumn{3}{|c|}{ pH } \\
\hline & $\begin{array}{c}1^{\mathrm{a}} \text { Coleta } \\
28-05-2008 \\
\end{array}$ & $\begin{array}{c}2^{\mathrm{a}} \text { Coleta } \\
25-11-2008 \\
\end{array}$ & $\begin{array}{l}3^{\text {a }} \text { Coleta } \\
30-07-09\end{array}$ \\
\hline 1 & 6,59 & 6,32 & 6,54 \\
\hline 2 & 6,33 & 6,20 & 6,25 \\
\hline 3 & 6,48 & 6,44 & 6,31 \\
\hline 4 & 6,45 & 6,20 & 6,19 \\
\hline 5 & 6,34 & 6,28 & 6,30 \\
\hline 6 & 6,40 & 6,58 & 6,22 \\
\hline 7 & 6,40 & 6,30 & 6,31 \\
\hline 8 & 5,78 & 5,77 & 5,80 \\
\hline 9 & 6,56 & 6,28 & 6,52 \\
\hline 10 & 6,30 & 6,40 & 6,18 \\
\hline 11 & 6,41 & 6,34 & 6,47 \\
\hline 12 & 6,51 & 6,36 & 6,41 \\
\hline 13 & 5,96 & 5,85 & 5,93 \\
\hline 14 & 6,40 & 6,20 & 6,81 \\
\hline 15 & 6,38 & 6,58 & 6,23 \\
\hline 16 & 6,48 & 6,63 & 6,70 \\
\hline 17 & 6,50 & 6,45 & 6,61 \\
\hline 18 & 5,60 & 6,22 & 6,10 \\
\hline 19 & 6,60 & 6,45 & 6,61 \\
\hline 20 & 6,59 & 6,48 & 6,34 \\
\hline 21 & 6,14 & 6,00 & 6,12 \\
\hline 22 & 6,48 & 6,37 & 6,35 \\
\hline 23 & 6,43 & 6,43 & 6,41 \\
\hline 24 & 6,45 & 6,29 & 6,00 \\
\hline 25 & 6,18 & 6,32 & 6,43 \\
\hline 26 & 6,00 & 6,03 & 6,00 \\
\hline 27 & 6,00 & 6,14 & 6,00 \\
\hline 28 & 5,82 & 5,71 & 5,82 \\
\hline 29 & 6,50 & 6,33 & 6,48 \\
\hline 30 & 5,56 & 5,64 & 5,50 \\
\hline 31 & 6,49 & 6,39 & 6,30 \\
\hline 32 & 6,00 & 5,93 & 6,95 \\
\hline 33 & 5,78 & 6,30 & 6,78 \\
\hline
\end{tabular}

\subsection{Curvas analíticas utilizadas para as determinações de metais}

As curvas analíticas foram construídas com 4 pontos. Na Figura 3, encontra-se a curva analítica para o Fe e na Tabela 5, os coeficientes de correlações para os metais analisados. 
SANCHES, S. M.; VIEIRA, E. M.; PRADO, E. L.; TAKAYANAGUI, A. M. M. Qualidade da água de abastecimento público de Ribeirão Preto em área de abrangência do Aquífero Guarani: determinação de metais e nitrato. Ambi-Agua, Taubaté, v. 5, n. 2, p. 202-216, 2010. (doi:10.4136/ambi-agua.148)

Tabela 4. Concentrações de nitrato em água de poços de abastecimento púbico de Ribeirão - SP.

\begin{tabular}{|c|c|c|c|}
\hline \multirow{2}{*}{$\begin{array}{c}\text { Poços de água de } \\
\text { abastecimento público de } \\
\text { Ribeirão Preto - SP }\end{array}$} & \multicolumn{3}{|c|}{ Concentração de nitrato $\left(\mathrm{mg} \mathrm{L}^{-1}\right)$} \\
\hline & $\begin{array}{c}1^{\mathrm{a}} \text { Coleta } \\
28-05-2008\end{array}$ & $\begin{array}{c}2^{\mathrm{a}} \text { Coleta } \\
25-11-2008\end{array}$ & $\begin{array}{c}3^{a} \text { Coleta } \\
30-07-2009\end{array}$ \\
\hline 1 & 0,0656 & 0,0615 & nd \\
\hline 2 & 3,6326 & 3,5612 & 3,9505 \\
\hline 3 & 0,0535 & 0,0173 & nd \\
\hline 4 & 3,3880 & 3,3100 & 3,2617 \\
\hline 5 & 1,7168 & 1,7984 & 1,6761 \\
\hline 6 & 2,7399 & 2,5954 & 2,4301 \\
\hline 7 & 0,4844 & 0,5277 & nd \\
\hline 8 & 0,3092 & 0,3870 & 0,1017 \\
\hline 9 & 0,0294 & 0,0214 & nd \\
\hline 10 & 0,0334 & 0,0280 & nd \\
\hline 11 & 0,0374 & 0,0050 & nd \\
\hline 12 & 0,0615 & 0,0254 & nd \\
\hline 13 & 1,0505 & 1,0028 & 0,8090 \\
\hline 14 & 0,0001 & 0,0133 & nd \\
\hline 15 & 0,1892 & 0,0414 & nd \\
\hline 16 & 0,1365 & 0,2022 & nd \\
\hline 17 & 0,0484 & 0,0655 & nd \\
\hline 18 & 0,0176 & 0,0294 & nd \\
\hline 19 & 0,0405 & 0,1098 & nd \\
\hline 20 & 0,0001 & nd & nd \\
\hline 21 & 0,7142 & 0,7728 & 0,5317 \\
\hline 22 & 0,0001 & nd & nd \\
\hline 23 & 0,0214 & nd & nd \\
\hline 24 & 0,0567 & 0,0467 & nd \\
\hline 25 & 0,5891 & 0,5397 & 0,2263 \\
\hline 26 & 0,0001 & nd & nd \\
\hline 27 & 0,6921 & 0,7407 & 0,6844 \\
\hline 28 & 0,0001 & nd & nd \\
\hline 29 & 3,4573 & 3,1000 & 2,7766 \\
\hline 30 & 0,4166 & 0,5397 & 0,2625 \\
\hline 31 & 0,0578 & 0,0468 & 0,0478 \\
\hline 32 & 0,0314 & 0,0414 & nd \\
\hline 33 & 0,0414 & nd & nd \\
\hline
\end{tabular}

nd - não detectável.

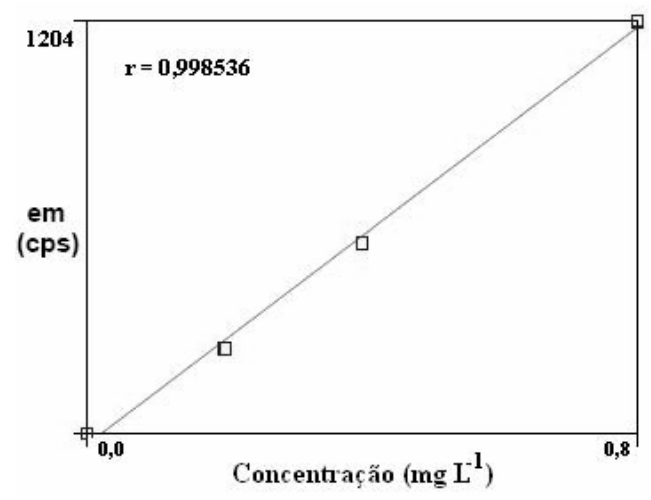

Figura 3. Curva analítica utilizada para a determinação de $\mathrm{Fe}$ em água de abastecimento público. 
Tabela 5. Valores dos coeficientes de correlação para as curvas analíticas dos metais.

\begin{tabular}{l|c}
\hline Metais & $\begin{array}{c}\text { Coeficientes } \\
\text { de correlação (r) }\end{array}$ \\
\hline Cromo & 0,999933 \\
Manganês & 0,996183 \\
Cádmio & 0,999946 \\
Cobre & 0,999927 \\
Zinco & 0,999906 \\
Ferro & 0,998536 \\
Chumbo & 0,998475 \\
\hline
\end{tabular}

Analisando-se os coeficientes de correlação, apresentandos na Tabela 5, observa-se que os coeficientes de correlação (R) foram superiores a 0,99 , indicando uma boa linearidade. A curva analítica obtida para os metais foram lineares, apresentando coeficientes de correlação (R) superiores a 0,99. A Agência Nacional de Vigilância Sanitária (Brasil, 2003) recomenda um coeficiente de correlação igual a 0,99 e o Instituto Nacional de Metrologia, Normalização e Qualidade Industrial (Brasil, 2003) acima de 0,90. Esse coeficiente de correlação foi satisfatório, o que demonstra que a resposta do detector foi linear nos intervalos de concentração empregados neste estudo.

\subsection{Metais}

Neste estudo foi determinada a concentração dos metais $\mathrm{Cr}, \mathrm{Cd}, \mathrm{Cu}, \mathrm{Zn}$, Fe e $\mathrm{Pb}$ em 33 poços de água de abastecimento público de Ribeirão Preto. Na Tabela 6 está apresentado o limite máximo permitido pela Port. MS nº 518/2004 (Brasil, 2004) para os metais.

Tabela 6. Valor máximo permitido pela Port. MS n ${ }^{0}$ 518/2004 para os metais analisados.

\begin{tabular}{|c|c|}
\hline Metais & $\begin{array}{l}\text { Valor Máximo Permitido } \\
\qquad\left(\mathrm{mg} \mathrm{L}^{-1}\right)\end{array}$ \\
\hline Cromo & 0,050 \\
\hline Manganês & 0,100 \\
\hline Cádmio & 0,005 \\
\hline Cobre & 2,000 \\
\hline Zinco & 5,000 \\
\hline Ferro & 0,300 \\
\hline Chumbo & 0,010 \\
\hline
\end{tabular}

Dentre alguns metais citados na Tabela 6, o Cd pode causar hipertensão, problemas nos rins e destruição dos glóbulos vermelhos. O Cr na forma hexavalente é considerado carcinogênico, podendo causar câncer no trato digestivo e pulmões (Boni e Sbaffoni, 2009). A toxicidade aguda por $\mathrm{Pb}$ provoca várias disfunções nos rins, no sistema reprodutivo, fígado, no cérebro e sistema nervoso central. Na Tabela 7, encontram-se os valores das concentrações dos metais para os poços de água de abastecimento público de Ribeirão Preto - SP, em três diferentes momentos de coleta. 
SANCHES, S. M.; VIEIRA, E. M.; PRADO, E. L.; TAKAYANAGUI, A. M. M. Qualidade da água de abastecimento público de Ribeirão Preto em área de abrangência do Aquífero Guarani: determinação de metais e nitrato. Ambi-Agua, Taubaté, v. 5, n. 2, p. 202-216, 2010. (doi:10.4136/ambi-agua.148)

Tabela 7. Concentrações de metais encontrados para a água de poços de abastecimento público.

\begin{tabular}{|c|c|c|c|c|c|c|c|}
\hline $\begin{array}{c}\text { Poços de água de } \\
\text { abastecimento público de } \\
\text { Ribeirão Preto - SP }\end{array}$ & $\begin{array}{c}\text { Data das } \\
\text { Coletas }\end{array}$ & $\mathrm{Cr}$ & Cd & $\mathrm{Cu}$ & Zn & $\mathbf{F e}$ & $\mathbf{P b}$ \\
\hline \multirow[t]{3}{*}{ 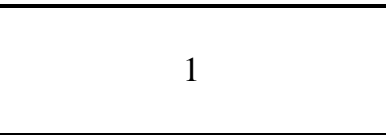 } & $28-05-2008$ & 0,0080 & nd & 0,390 & nd & nd & nd \\
\hline & $25-11-2008$ & $\mathrm{Nd}$ & nd & nd & 0,008 & 0,035 & nd \\
\hline & $30-07-2009$ & 0,0009 & 0,0020 & 0,025 & 0,032 & 0,057 & nd \\
\hline \multirow{3}{*}{2} & $28-05-2008$ & $\mathrm{Nd}$ & nd & 0,280 & nd & nd & nd \\
\hline & 25-11-2008 & $\mathrm{Nd}$ & nd & nd & nd & 0,029 & nd \\
\hline & $30-07-2009$ & 0,0019 & 0,0003 & 0,021 & 0,024 & 0,041 & nd \\
\hline \multirow{3}{*}{3} & $28-05-2008$ & $\mathrm{Nd}$ & 0,0010 & nd & nd & nd & nd \\
\hline & $25-11-2008$ & 0,0010 & nd & nd & 0,005 & 0,013 & nd \\
\hline & $30-07-2009$ & 0,0019 & 0,0003 & 0,021 & 0,023 & 0,056 & nd \\
\hline \multirow{3}{*}{4} & $28-05-2008$ & 0,010 & nd & 0,400 & nd & 0,140 & nd \\
\hline & $25-11-2008$ & 0,0020 & nd & 0,335 & nd & 0,032 & nd \\
\hline & $30-07-2009$ & 0,0020 & 0,0005 & 0,018 & 0,028 & 0,053 & nd \\
\hline \multirow{3}{*}{5} & $28-05-2008$ & 0,0200 & nd & 0,340 & nd & nd & nd \\
\hline & 25-11-2008 & Nd & nd & 0,070 & nd & 0,025 & nd \\
\hline & $30-07-2009$ & 0,0035 & 0,0003 & 0,018 & 0,042 & 0,049 & nd \\
\hline \multirow{3}{*}{6} & $28-05-2008$ & 0,0010 & nd & 0,030 & nd & 0,020 & nd \\
\hline & $25-11-2008$ & 0,0030 & nd & nd & 0,004 & 0,039 & nd \\
\hline & $30-07-2009$ & 0,0016 & 0,0004 & 0,018 & 0,024 & 0,054 & nd \\
\hline \multirow{3}{*}{7} & $28-05-2008$ & 0,040 & nd & 0,310 & 0,200 & nd & nd \\
\hline & $25-11-2008$ & $\mathrm{Nd}$ & nd & 0,120 & nd & 0,023 & nd \\
\hline & $30-07-2009$ & 0,0009 & 0,0006 & 0,021 & 0,003 & 0,024 & $\mathrm{Nd}$ \\
\hline \multirow{3}{*}{8} & $28-05-2008$ & $\mathrm{Nd}$ & nd & 0,330 & nd & nd & nd \\
\hline & $25-11-2008$ & $\mathrm{Nd}$ & nd & 0,040 & nd & 0,120 & nd \\
\hline & $30-07-2009$ & 0,0017 & 0,0005 & 0,017 & 0,009 & 0,023 & nd \\
\hline \multirow{3}{*}{9} & $28-05-2008$ & 0,0050 & nd & 0,020 & nd & 0,019 & nd \\
\hline & $25-11-2008$ & 0,0070 & nd & nd & nd & 0,027 & nd \\
\hline & $30-07-2009$ & 0,0006 & 0,0004 & 0,016 & 0,088 & 0,052 & nd \\
\hline \multirow{3}{*}{10} & $28-05-2008$ & $\mathrm{Nd}$ & nd & 0,330 & nd & nd & nd \\
\hline & $25-11-2008$ & $\mathrm{Nd}$ & nd & 0,250 & nd & nd & nd \\
\hline & $30-07-2009$ & 0,0010 & 0,0004 & 0,017 & 0,021 & 0,026 & nd \\
\hline \multirow{3}{*}{11} & 28-05-2008 & nd & nd & 0,380 & 0,320 & nd & nd \\
\hline & $25-11-2008$ & nd & nd & 0,360 & nd & 0,038 & nd \\
\hline & $30-07-2009$ & 0,0016 & 0,0005 & 0,015 & 0,040 & 0,026 & nd \\
\hline \multirow{3}{*}{12} & 28-05-2008 & nd & nd & nd & nd & nd & nd \\
\hline & $25-11-2008$ & nd & nd & nd & 0,027 & 0,044 & nd \\
\hline & $30-07-2009$ & 0,0018 & 0,0004 & 0,018 & 0,011 & 0,041 & nd \\
\hline \multirow{3}{*}{13} & $28-05-2008$ & 0,0200 & nd & 0,760 & nd & nd & nd \\
\hline & $25-11-2008$ & 0,0070 & nd & nd & 0,036 & 0,093 & nd \\
\hline & $30-07-2009$ & 0,0010 & 0,0005 & 0,019 & nd & 0,021 & nd \\
\hline \multirow{3}{*}{14} & $28-05-2008$ & 0,010 & nd & 0,410 & 0,260 & nd & nd \\
\hline & $25-11-2008$ & 0,004 & nd & nd & 0,090 & nd & nd \\
\hline & $30-07-2009$ & 0,001 & 0,0007 & 0,023 & 0,046 & 0,051 & nd \\
\hline \multirow{3}{*}{15} & 28-05-2008 & nd & nd & nd & 0,019 & 0,017 & nd \\
\hline & $25-11-2008$ & nd & nd & nd & 0,029 & 0,023 & nd \\
\hline & $30-07-2009$ & 0,0009 & 0,0007 & 0,019 & 0,014 & 0,037 & nd \\
\hline \multirow{3}{*}{16} & $28-05-2008$ & 0,070 & nd & 0,380 & nd & nd & nd \\
\hline & $25-11-2008$ & nd & nd & nd & nd & 0,035 & nd \\
\hline & $30-07-2009$ & 0,0006 & 0,0005 & 0,019 & 0,025 & 0,031 & nd \\
\hline \multirow{3}{*}{17} & $28-05-2008$ & nd & nd & 0,280 & nd & 0,050 & nd \\
\hline & $25-11-2008$ & nd & nd & nd & nd & 0,039 & nd \\
\hline & $30-07-2009$ & 0,0015 & 0,0005 & 0,018 & nd & 0,048 & nd \\
\hline
\end{tabular}


SANCHES, S. M.; VIEIRA, E. M.; PRADO, E. L.; TAKAYANAGUI, A. M. M. Qualidade da água de abastecimento público de Ribeirão Preto em área de abrangência do Aquífero Guarani: determinação de metais e nitrato. Ambi-Agua, Taubaté, v. 5, n. 2, p. 202-216, 2010. (doi:10.4136/ambi-agua.148)

Continuação

\begin{tabular}{|c|c|c|c|c|c|c|c|}
\hline 18 & $\begin{array}{l}28-05-2008 \\
25-11-2008 \\
30-07-2009 \\
\end{array}$ & $\begin{array}{c}0,0300 \\
\text { nd } \\
0,0013\end{array}$ & $\begin{array}{c}\text { nd } \\
\text { nd } \\
0,0006 \\
\end{array}$ & $\begin{array}{l}0,370 \\
0,090 \\
0,017\end{array}$ & $\begin{array}{c}\text { nd } \\
\text { nd } \\
0.021\end{array}$ & $\begin{array}{c}\text { nd } \\
0,025 \\
0,021\end{array}$ & $\begin{array}{l}\text { nd } \\
\text { nd } \\
\text { nd }\end{array}$ \\
\hline 19 & $\begin{array}{l}28-05-2008 \\
25-11-2008 \\
30-07-2009\end{array}$ & $\begin{array}{c}\text { nd } \\
\text { nd } \\
0,0008\end{array}$ & $\begin{array}{c}\text { nd } \\
\text { nd } \\
0,0006\end{array}$ & $\begin{array}{c}0,320 \\
\text { nd } \\
0,018\end{array}$ & $\begin{array}{c}\text { nd } \\
0,004 \\
0,034\end{array}$ & $\begin{array}{c}\text { nd } \\
0,038 \\
0,022\end{array}$ & $\begin{array}{l}\text { nd } \\
\text { nd } \\
\text { nd }\end{array}$ \\
\hline 20 & $\begin{array}{l}28-05-2008 \\
25-11-2008 \\
30-07-2009\end{array}$ & $\begin{array}{l}0,0020 \\
0,0050 \\
0,0018\end{array}$ & $\begin{array}{c}\text { nd } \\
\text { nd } \\
0,0005\end{array}$ & $\begin{array}{c}\text { nd } \\
\text { nd } \\
0,013\end{array}$ & $\begin{array}{l}\text { nd } \\
\text { nd } \\
\text { nd }\end{array}$ & $\begin{array}{l}0,003 \\
0,009 \\
0,028\end{array}$ & $\begin{array}{l}\text { nd } \\
\text { nd } \\
\text { nd }\end{array}$ \\
\hline 21 & $\begin{array}{l}28-05-2008 \\
25-11-2008 \\
30-07-2009\end{array}$ & $\begin{array}{c}\mathrm{Nd} \\
\mathrm{Nd} \\
0,0012\end{array}$ & $\begin{array}{c}\text { nd } \\
\text { nd } \\
0,0007\end{array}$ & $\begin{array}{c}0,390 \\
\text { nd } \\
0,022\end{array}$ & $\begin{array}{c}\text { nd } \\
\text { nd } \\
0.036\end{array}$ & $\begin{array}{l}0,260 \\
0,022 \\
0,054\end{array}$ & $\begin{array}{l}\text { nd } \\
\text { nd } \\
\text { nd }\end{array}$ \\
\hline 22 & $\begin{array}{l}28-05-2008 \\
25-11-2008 \\
30-07-2009\end{array}$ & $\begin{array}{c}\mathrm{Nd} \\
\mathrm{Nd} \\
0,0007\end{array}$ & $\begin{array}{c}\text { nd } \\
\text { nd } \\
0,0005\end{array}$ & $\begin{array}{c}0,320 \\
\text { nd } \\
0,021\end{array}$ & $\begin{array}{c}\text { nd } \\
\text { nd } \\
0,005\end{array}$ & $\begin{array}{c}\text { nd } \\
0,043 \\
0,014\end{array}$ & $\begin{array}{l}\text { nd } \\
\text { nd } \\
\text { nd }\end{array}$ \\
\hline 23 & $\begin{array}{l}28-05-2008 \\
25-11-2008 \\
30-07-2009\end{array}$ & $\begin{array}{c}0,010 \\
\mathrm{Nd} \\
0.0013\end{array}$ & $\begin{array}{c}\text { nd } \\
\text { nd } \\
0,0005\end{array}$ & $\begin{array}{c}0,350 \\
\text { nd } \\
0,021\end{array}$ & $\begin{array}{c}\text { nd } \\
\text { nd } \\
0,044\end{array}$ & $\begin{array}{c}\text { nd } \\
0,017 \\
0,041\end{array}$ & $\begin{array}{l}\text { nd } \\
\text { nd } \\
\text { nd }\end{array}$ \\
\hline 24 & $\begin{array}{l}28-05-2008 \\
25-11-2008 \\
30-07-2009\end{array}$ & $\begin{array}{c}\mathrm{Nd} \\
\mathrm{Nd} \\
0,0006\end{array}$ & $\begin{array}{c}\text { nd } \\
\text { nd } \\
0,0007\end{array}$ & $\begin{array}{l}0,110 \\
0,060 \\
0,023\end{array}$ & $\begin{array}{c}\text { nd } \\
\text { nd } \\
0,002\end{array}$ & $\begin{array}{c}\text { nd } \\
\text { nd } \\
0,022\end{array}$ & $\begin{array}{l}\text { nd } \\
\text { nd } \\
\text { nd }\end{array}$ \\
\hline 25 & $\begin{array}{l}28-05-2008 \\
25-11-2008 \\
30-07-2009\end{array}$ & $\begin{array}{l}\mathrm{Nd} \\
\mathrm{Nd} \\
\mathrm{Nd}\end{array}$ & $\begin{array}{c}\text { nd } \\
\text { nd } \\
0,0007\end{array}$ & $\begin{array}{l}0,340 \\
0,190 \\
0,014\end{array}$ & $\begin{array}{c}\text { nd } \\
\text { nd } \\
0,011\end{array}$ & $\begin{array}{c}\text { nd } \\
\text { nd } \\
0,017\end{array}$ & $\begin{array}{l}\text { nd } \\
\text { nd } \\
\text { nd }\end{array}$ \\
\hline 26 & $\begin{array}{l}28-05-2008 \\
25-11-2008 \\
30-07-2009 \\
\end{array}$ & $\begin{array}{c}\mathrm{Nd} \\
\mathrm{Nd} \\
0,0006 \\
\end{array}$ & $\begin{array}{c}\text { nd } \\
\text { nd } \\
0,0005\end{array}$ & $\begin{array}{l}0,260 \\
0,261 \\
0,019\end{array}$ & $\begin{array}{c}\text { nd } \\
0,052 \\
0,020 \\
\end{array}$ & $\begin{array}{l}0,020 \\
0,036 \\
0,040\end{array}$ & $\begin{array}{l}\text { nd } \\
\text { nd } \\
\text { nd }\end{array}$ \\
\hline 27 & $\begin{array}{l}28-05-2008 \\
25-11-2008 \\
30-07-2009\end{array}$ & $\begin{array}{c}0,040 \\
0,010 \\
0,0009\end{array}$ & $\begin{array}{c}\text { nd } \\
\text { nd } \\
0,0004\end{array}$ & $\begin{array}{l}0,390 \\
0,170 \\
0,018\end{array}$ & $\begin{array}{c}\text { nd } \\
\text { nd } \\
0,076\end{array}$ & $\begin{array}{c}0,051 \\
\text { nd } \\
0,045\end{array}$ & $\begin{array}{l}\text { nd } \\
\text { nd } \\
\text { nd }\end{array}$ \\
\hline 28 & $\begin{array}{l}28-05-2008 \\
25-11-2008 \\
30-07-2009 \\
\end{array}$ & $\begin{array}{c}\mathrm{Nd} \\
\mathrm{Nd} \\
0,0011\end{array}$ & $\begin{array}{c}\text { nd } \\
\text { nd } \\
0,0004\end{array}$ & $\begin{array}{l}0,200 \\
0,082 \\
0,016\end{array}$ & $\begin{array}{c}\text { nd } \\
\text { nd } \\
0,031\end{array}$ & $\begin{array}{c}\text { nd } \\
0,015 \\
0,048 \\
\end{array}$ & $\begin{array}{l}\text { nd } \\
\text { nd } \\
\text { nd }\end{array}$ \\
\hline 29 & $\begin{array}{l}28-05-2008 \\
25-11-2008 \\
30-07-2009 \\
\end{array}$ & $\begin{array}{c}\mathrm{Nd} \\
\mathrm{Nd} \\
0,0018 \\
\end{array}$ & $\begin{array}{c}\text { nd } \\
\text { nd } \\
0,0006 \\
\end{array}$ & $\begin{array}{c}0,290 \\
\text { nd } \\
0,016 \\
\end{array}$ & $\begin{array}{c}\text { nd } \\
\text { nd } \\
0,024 \\
\end{array}$ & $\begin{array}{l}0,010 \\
0,069 \\
0,049 \\
\end{array}$ & $\begin{array}{l}\text { nd } \\
\text { nd } \\
\text { nd }\end{array}$ \\
\hline 30 & $\begin{array}{l}28-05-2008 \\
25-11-2008 \\
30-07-2009\end{array}$ & $\begin{array}{c}\mathrm{Nd} \\
\mathrm{Nd} \\
0,0013\end{array}$ & $\begin{array}{c}\text { nd } \\
\text { nd } \\
0,0005\end{array}$ & $\begin{array}{l}0,330 \\
0,360 \\
0,014\end{array}$ & $\begin{array}{c}\text { nd } \\
0,001 \\
\text { nd }\end{array}$ & $\begin{array}{c}0,24 \\
0,035 \\
0,020\end{array}$ & $\begin{array}{l}\text { nd } \\
\text { nd } \\
\text { nd }\end{array}$ \\
\hline 31 & $\begin{array}{l}28-05-2008 \\
25-11-2008 \\
30-07-2009\end{array}$ & $\begin{array}{c}0,02 \\
\mathrm{Nd} \\
0,0012\end{array}$ & $\begin{array}{c}\text { nd } \\
\text { nd } \\
0,0005\end{array}$ & $\begin{array}{c}0,16 \\
0,100 \\
0,016\end{array}$ & $\begin{array}{c}\text { nd } \\
\text { nd } \\
0,058\end{array}$ & $\begin{array}{l}0,240 \\
0,110 \\
0,034\end{array}$ & $\begin{array}{l}\text { nd } \\
\text { nd } \\
\text { nd }\end{array}$ \\
\hline 32 & $\begin{array}{l}28-05-2008 \\
25-11-2008 \\
30-07-2009\end{array}$ & $\begin{array}{c}\mathrm{Nd} \\
\mathrm{Nd} \\
0,0012\end{array}$ & $\begin{array}{c}\text { nd } \\
\text { nd } \\
0,0005\end{array}$ & $\begin{array}{c}0,310 \\
\text { nd } \\
0,016\end{array}$ & $\begin{array}{c}\text { nd } \\
\text { nd } \\
0,058\end{array}$ & $\begin{array}{c}\text { nd } \\
0,015 \\
0,034\end{array}$ & $\begin{array}{l}\text { nd } \\
\text { nd } \\
\text { nd }\end{array}$ \\
\hline 33 & $\begin{array}{l}28-05-2008 \\
25-11-2008 \\
30-07-2009\end{array}$ & $\begin{array}{c}0,031 \\
\mathrm{Nd} \\
0,0024\end{array}$ & $\begin{array}{c}\text { nd } \\
\text { nd } \\
0,0004\end{array}$ & $\begin{array}{c}0,270 \\
\text { nd } \\
0,013\end{array}$ & $\begin{array}{c}\text { nd } \\
0,030 \\
\text { nd }\end{array}$ & $\begin{array}{l}0,040 \\
0,059 \\
0,026\end{array}$ & $\begin{array}{l}\text { nd } \\
\text { nd } \\
\text { nd }\end{array}$ \\
\hline
\end{tabular}


Analisando-se os resultados apresentados na Tabela 7, e comparando-os com os valores determinados pela Port. MS $n^{\circ}$ 518/2004 (Tabela 6), observa-se que não foi ultrapassado o limite máximo permitido dos metais analisados para a água analisada dos 33 poços de água de abastecimento público. Cabe ressaltar que a presença dos metais $\mathrm{Fe}, \mathrm{Zn}$ e $\mathrm{Cu}$ devem-se, principalmente, ao material no qual foram construídas as tubulações dos poços de água de abastecimento (alguns foram de ferro galvanizado e outros de cobre). Já a presença de Cr na água subterrânea pode estar relacionada à contribuição de rochas, que são fontes naturais. Sabe-se que o cromo é disponibilizado naturalmente pela lixiviação dos minerais contendo cromo, cromita (Cetesb, 2006). Segundo o relatório de qualidade de águas subterrâneas no estado de São Paulo, com exceção do Aquífero Bauru, que apresenta um valor de referência de qualidade de cromo total de 0,003, obteve-se para os demais um valor de referência da ordem de 0,001 a $0,005 \mathrm{mg} \mathrm{L}^{-1}$.

\section{CONCLUSÃO}

A partir das análises de amostras de água de 33 poços de abastecimento público de Ribeirão Preto-SP, selecionadas nesta investigação, nos meses de maio e novembro de 2008, e julho de 2009 realizados em 3 diferentes períodos, pode-se concluir que:

Quanto aos parâmetros metais e nitrato, todas as amostras apresentaram-se dentro dos valores estabelecidos pela Portaria MS no 518/2004 (Brasil, 2004);

Em relação ao $\mathrm{pH}$, um pequeno número de poços de abastecimento público apresentou $\mathrm{pH}$ fora do limite de potabilidade, porém valores muito próximos do limite inferior da Portaria 518 do Ministério da Saúde de 2004.

A água dos poços de abastecimento público de Ribeirão Preto - SP apresentou, de uma forma geral, uma boa qualidade, não apresentando praticamente nenhuma restrição de uso, exceto quanto ao $\mathrm{pH}$. Essa característica foi comprovada pelas análises químicas, cujos valores de uma forma geral estão dentro do padrão de aceitação para o consumo humano quanto aos parâmetros estudados.

Pelos resultados considera-se ser necessário um contínuo monitoramento de todos os poços de abastecimento público, especialmente quanto à presença de nitrato e cromo, porque segundo o Relatório de Qualidade das Águas Subterrâneas do Estado de São Paulo, publicado pela Cetesb entre 2004 e 2006, há uma tendência no aumento, em situações de abastecimento público, o que representa um risco a saúde pública.

\section{AGRADECIMENTOS}

À Fundação de Amparo à pesquisa do Estado de São Paulo - FAPESP, pelo apoio concedido para a execução deste trabalho.

\section{REFERÊNCIAS}

ALABURDA, J.; NISHIHARA, L. Presença de compostos de nitrogênio em águas de poços. Revista de Saúde Pública, v. 32, p. 160 - 165, 1998.

BONI, M. R.; SBAFFONI, S. The potential of compost-based biobarriers for Cr(VI) removal from contaminated groundwater: Column test. Journal of Hazardous Materials, v. 166, p. 1087 - 1095, 2009.

BORGHETTI, N. R. B.; BORGHETTI, J. R.; ROSA FILHO, E. F. Aquifero Guarani: a verdadeira integração dos países do Mercosul. Curitiba: CDU, 204. 214p. 
BOUCHARD, D. C.; WILLIAM, S. M. K. Nitrate contamination of groundwater; sources and potential health effects. Journal of the American Water Works Association, v. 9, p. 85- 90, 1992.

BRASIL. Ministério da Saúde. Agência Nacional de Vigilância Sanitária - ANVISA. Resolução RE n. 899 de 29 de maio de 2003. Disponível em: $<$ http://www.anvisa.gov.br/legis/resol/2003/re/899_03re.htm>. Acesso em: 20 de dezembro de 2008.

BRASIL. Ministério da Saúde. Secretaria de Vigilância em Saúde. Coordenação-Geral de Vigilância em Saúde Ambiental. Portaria n 518/2004, de 25 de março de 2004: estabelece os procedimentos e responsabilidades relativas ao controle de vigilância da qualidade da água para o consumo humano e seu padrão de potabilidade, e dá outras providências. Disponível em: <http://portal.saude.gov.br/portal/arquivos/pdf/portaria _518_2004.pdf>. Acesso em: 30 de julho de 2009.

CELERE, M. S; OLIVEIRA, A. S.; TREVILATO, T. M. B.; SEGURA-MUÑOZ, S. I. Metais presentes no chorume coletado no aterro sanitário de Ribeirão Preto, São Paulo, Brasil, e sua relevância para saúde pública. Caderno de Saúde Pública, v. 23, n. 4, p. 939 947, 2007.

COMPANHIA DE TECNOLOGIA E SANEAMENTO AMBIENTAL - CETESB. Variáveis de qualidade das águas. 2001. Disponível em: <http://www.cetesb.sp.gov.br/Agua/ rios/variaveis.asp\#zinco> Acesso em: 12 de novembro de 2008.

ECKHARDT, R. R.; DIEDRICH, V. L.; FERREIRA, E. R.; STROHSCHOEN, E.; DEMAMAN, L. C. Mapeamento e avaliação da potabilidade da água subterrânea do município de Lajeado, RS, Brasil. Ambi-Agua, Taubaté, v. 4, n. 1, p. 58 - 80, 2009.

GASTAMANS, D.; KIANG, C. H. Avaliação da hidrogeologia e hidroquímica do sistema Aqüifero Guarani no estado do Mato Grosso do Sul. Revista Águas subterrâneas, São Paulo, v. 19, n. 1, p. 35 - 48, 2005.

IRITANI, M. A.; EZAKI, S. As águas subterrâneas do Estado de São Paulo. São Paulo: Secretaria do Estado do Meio Ambiente, 2008. 104p.

MACÊDO, J. A. B. Águas \& Águas. Belo Horizonte: CRQ-MG, 2004. 977p.

Rebouças, A. C. Impactos ambientais nas águas subterrâneas. In: CONGRESSO BRASILEIRO DE ÁGUAS SUBTERRÂNEAS, 7., 1992, Belo Horizonte. Anais...

Belo Horizonte: ABAS, 1992. p. 11 - 17.

REBOUÇAS, A. C.; BRAGA, B.; TUNDISU, J. G. (Orgs.) Águas doces no Brasil: capital ecológico, uso e conservação. 3. ed. São Paulo: Escrituras, 2006. 749p.

RIBEIRÃO PRETO (Município). Departamento de Água e Esgoto de Ribeirão Preto (SP) DAERP. 2007. Disponível em: <http://www.ribeiraopretosp.gov.br> Acesso em: 20 de agosto de 2009.

SILVA, R. B. G. Águas subterrâneas: um valioso recurso que requer proteção. São Paulo: DAEE, 2003. 27p. 
SANCHES, S. M.; VIEIRA, E. M.; PRADO, E. L.; TAKAYANAGUI, A. M. M. Qualidade da água de abastecimento público de Ribeirão Preto em área de abrangência do Aquífero Guarani: determinação de metais e nitrato. Ambi-Agua, Taubaté, v. 5, n. 2, p. 202-216, 2010. (doi:10.4136/ambi-agua.148)

TONANI, K. A. A. Identificação e quantificação de metais pesados, parasitas e bactérias em esgoto bruto e tratado da Estação de Tratamento de Esgoto de Ribeirão Preto. 2008. 179f. Dissertação (Mestrado em Saúde Pública) - Escola de Enfermagem de Ribeirão Preto, Universidade de São Paulo, São Paulo, 2008.

UNITED STATES ENVIRONMENTAL PROTECTION AGENCY - USEPA. Acid digestion of waters for total recoverable or dissolved metals for analysis by FLAA or ICP Spectroscopy. 1992. Disponível em: <http://www.epa.gov/epaoswer/hazwaste/ test/3_series.htm>. Acesso em: 15 maio 2009. 